\title{
Bayesian Early Mode Decision Technique for View Synthesis Prediction-Enhanced Multiview Video Coding
}

\author{
Shadan Khattak, Raouf Hamzaoui, Thomas Maugey, Shakeel Ahmad, and Pascal Frossard
}

\begin{abstract}
View synthesis prediction (VSP) is a coding mode that predicts video blocks from synthesised frames. It is particularly useful in a multi-camera setup with large inter-camera distances. Adding a VSP-based SKIP mode to a standard Multiview Video Coding (MVC) framework improves the rate-distortion (RD) performance but increases the time complexity of the encoder. This letter proposes an early mode decision technique for VSP SKIP-enhanced MVC. Our method uses the correlation between the RD costs of the VSP SKIP mode in neighbouring views and Bayesian decision theory to reduce the number of candidate coding modes for a given macroblock. Simulation results showed that our technique can save up to $36.20 \%$ of the encoding time without any significant loss in RD performance.
\end{abstract}

Index Terms - Fast mode decision, multiview video coding, view synthesis prediction.

\section{INTRODUCTION}

$\mathbf{T}$ HE multiview plus depth representation is one of the most promising methods for providing multiview video services [1]. In a multiview plus depth representation, the information consists of multiple texture views together with their associated per-pixel depth maps. View synthesis uses the per-pixel depth maps and interpolation techniques to synthesize virtual views between camera views. Traditionally, it has been used to reduce the network and storage resource consumption of multiview video by providing $N$ views (camera views plus synthesized views) at the decoder side while only $K(K<N)$ camera views are captured, encoded, and transmitted. However, view synthesis can also improve the RD performance of MVC by providing new prediction modes for blocks to be encoded [2]. In particular, a VSP-based SKIP mode has been shown [3] to significantly improve the RD performance of MVC. Unlike the conventional SKIP mode, the

Manuscript received May 28, 2013; revised August 10, 2013; accepted August 22, 2013. Date of publication September 16, 2013; date of current version September 25, 2013. The associate editor coordinating the review of this manuscript and approving it for publication was Prof. Yiannis Andreopoulos.

S. Khattak, R. Hamzaoui, and S. Ahmad are with the Faculty of Technology, De Montfort University, Leicester, LE1 9BH, U.K. (e-mail: shadan. khattak@myemail.dmu.ac.uk,rhamzaoui@dmu.ac.uk; sahmad@dmu.ac.uk).

T. Maugey and P. Frossard are with the Signal Processing Laboratory (LTS4), Ecole Polytechnique Fédérale de Lausanne (EPFL), 1015 Lausanne, Switzerland (e-mail: thomas.maugey@epfl.ch; pascal.frossard@epfl.ch).

Color versions of one or more of the figures in this paper are available online at http://ieeexplore.ieee.org.

Digital Object Identifier 10.1109/LSP.2013.2281607
VSP SKIP mode predicts a macroblock using a synthetic reference frame. However, the RD optimized framework of MVC already uses a computationally complex motion and disparity estimation process. Adding the VSP SKIP mode in this framework further increases the computational complexity of the encoding.

In this letter, we propose an early mode selection technique to reduce the time complexity of a VSP SKIP-enhanced MVC coder. We exploit the inter-view correlation between the RD costs of the VSP SKIP mode and use Bayesian decision theory to restrict the number of candidate coding modes that are tested during the encoding. In this way, motion and disparity estimation can be skipped for a large proportion of macroblocks. Our results show that the encoding time can be reduced by up to $36.20 \%$ compared to the latest version of the MVC Joint Multiview Video Coding (JMVC) reference software with integrated VSP SKIP mode, without any significant penalty on the RD performance.

No previous work has specifically addressed the problem of early mode selection for VSP SKIP-enhanced MVC. Most of the related work has focused on improving the quality of view synthesis [4], generating better depth maps [5], or pre- and postprocessing of synthesized images for better prediction [6]. A number of fast algorithms [7]-[12] have been proposed to reduce the time complexity of motion estimation, disparity estimation, reference frame selection, and mode decision processes in MVC. An early prediction technique for the Conventional SKIP mode in MVC was proposed in [13]. The method selects the Conventional SKIP mode for a macroblock if the corresponding macroblock identified by the Global Disparity Vector (GDV) [14] and its eight neighbouring macroblocks in a neighbouring view are encoded using this mode.

Bayesian decision theory was previously used for fast mode decision of H.264/AVC in [15] and Scalable Video Coding (SVC) in [16]. The techniques presented in these papers are not suitable for the proposed VSP-SKIP enhanced MVC coder as they do not exploit inter-view correlation. Moreover, our technique is unique in its use of the RD cost of a mode as the observed feature in the Bayesian decision rule.

The remainder of the letter is organized as follows. Section II presents the VSP SKIP-enhanced MVC coder considered in this letter and studies optimal coding modes for this coder. Section III proposes our Bayesian early mode decision technique. Section IV evaluates the performance of our method in terms of encoding time, bitrate, and peak signal-to-noise 


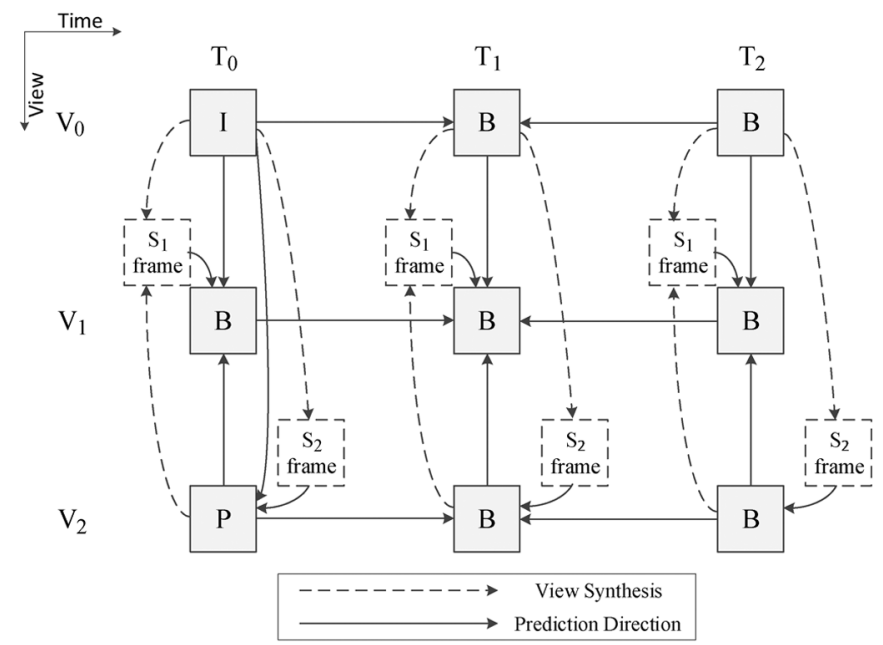

Fig. 1. Prediction structure using view synthesis. $V_{0}, V_{1}$, and $V_{2}$ are camera views while $S_{1}$ and $S_{2}$ are synthesized views. Dotted lines represent the reference view(s) for view synthesis and solid lines refer to the prediction direction. The synthesized frames are used as reference frames for VSP prediction.

ratio (PSNR) and compares it to a baseline approach based on inter-view mode correlation. Conclusions are given in Section V.

\section{PRELIMINARIES}

In this letter, we consider an extended MVC coder where a VSP SKIP mode is added to the existing eight Inter modes (Conventional SKIP, Inter $16 \times 16$, Inter $16 \times 8$, Inter8 $\times 16$, Inter8 $\times 8$, Inter $8 \times 4$, Inter $4 \times 8$, and Inter $4 \times 4$ ) and two Intra modes (Intra16 and Intra4) [10]. An example of the encoding structure with three camera views, $V_{0}, V_{1}$, and $V_{2}$, and two synthesized views $S_{1}$ and $S_{2}$ is illustrated in Fig. 1.

Conventional SKIP is a special mode in which the macroblock is normally reconstructed by motion-compensated prediction using a motion vector that is derived as the median value of the motion vectors of the left, top and top-right macroblocks [17]. The VSP SKIP mode differs from the conventional SKIP mode in that the macroblock is reconstructed using the macroblock at the same position in a synthesized version of the current frame [2].

To synthesize the views required for VSP SKIP, we use the method proposed in [18], which is based on the image coordinate system, the camera coordinate system, and the world coordinate system. A pixel in the image coordinate system of the camera view is projected onto a pixel in the image coordinate system of the virtual view in two steps. First, using the intrinsic and extrinsic parameters of the reference camera and the depth information, the 3D point that corresponds to the pixel in the camera view is projected onto the world coordinate system. Then, from the world coordinate system it is projected onto the image coordinate system of the virtual view (using the camera parameters of the virtual view). When switching viewpoints, some background regions which are hidden behind foreground objects in the reference view, might appear in the virtual view and vice versa. This induces the hole problem. When a synthesized frame is created using only one reference frame, holes cannot be efficiently filled. This problem is solved by using two reference frames where the second reference frame is used to fill the holes.
TABLE I

PROPORTION (\%) OF CODING MODES FOR MACROBLOCKS

\begin{tabular}{lcccccr}
\hline & \multicolumn{7}{c}{ Breakdancers sequence } \\
\hline Mode $\backslash$ QP (Texture) & 36 & 32 & 28 & 24 & 20 & Avg. \\
\hline VSP SKIP & 40.36 & 34.50 & 28.82 & 24.69 & 20.16 & 29.70 \\
\hline Conventional SKIP & 45.48 & 46.82 & 46.09 & 41.98 & 32.83 & 42.64 \\
\hline Inter16x16 & 11.60 & 14.37 & 17.73 & 20.62 & 23.64 & 17.59 \\
\hline Inter16x8 & 0.96 & 1.63 & 2.49 & 4.23 & 6.44 & 3.15 \\
\hline Inter8x16 & 0.97 & 1.46 & 2.75 & 4.33 & 6.19 & 3.14 \\
\hline Inter8x8 & 0.59 & 1.14 & 1.96 & 3.58 & 7.7 & 2.99 \\
\hline Intra modes & 0.04 & 0.08 & 0.16 & 0.58 & 3.25 & 0.82 \\
\hline & & \multicolumn{5}{c}{ Ballet sequence } \\
\hline Mode\QP (Texture) & 36 & 32 & 28 & 24 & 20 & Avg. \\
\hline VSP SKIP & 29.91 & 26.54 & 23.46 & 20.76 & 18.28 & 23.79 \\
\hline Conventional SKIP & 64.30 & 65.66 & 66.41 & 65.97 & 61.86 & 64.84 \\
\hline Inter16x16 & 4.80 & 6.16 & 7.61 & 9.38 & 13.68 & 8.33 \\
\hline Inter16x8 & 0.45 & 0.72 & 1.10 & 1.51 & 2.17 & 1.19 \\
\hline Inter8x16 & 0.36 & 0.60 & 0.91 & 1.48 & 2.25 & 1.12 \\
\hline Inter8x8 & 0.19 & 0.29 & 0.47 & 0.83 & 1.56 & 0.66 \\
\hline Intra modes & 0.00 & 0.03 & 0.03 & 0.08 & 0.20 & 0.06 \\
\hline
\end{tabular}

Table I shows the proportion of modes (at the macroblock level) selected for view $V_{1}$ in the settings of Fig. 1 and for a VSP SKIP-enhanced JMVC 6.0 reference software [19]. We used two test sequences (Breakdancers and Ballet [20]) of resolution $1024 \times 768$ and five quantization parameter (QP) values $(20,24,28,32,36)$. The number of frames in each sequence is 100 , the Group of Pictures (GOP) size is 16 , and the search range is $[ \pm 16, \pm 16]$. In the table, Inter $8 \times 8$ includes macroblocks encoded using sub modes Inter $8 \times 4$, Inter $4 \times 8$, and Inter4 $\times 4$. We observe that VSP SKIP and conventional SKIP are the dominant modes. The dominance is more prominent in the presence of large homogeneous regions (as in the Ballet sequence). We also observe that the other modes are rarely used.

\section{BAYESIAN EARly Mode Decision TECHNIQUE}

In the standard encoding scheme, the encoder considers all coding modes and selects one with minimum RD cost. The reference views ( $V_{0}$ and $V_{2}$ in Fig. 1) are encoded first, followed by the bidirectionally predicted view ( $V_{1}$ in Fig. 1). However, as observed in Section II, VSP SKIP or Conventional SKIP may be selected much more often than the other modes. In this section, we propose to exploit the correlation between VSP SKIP $\mathrm{RD}$ costs across views and Bayesian decision theory to avoid testing unlikely coding modes during the encoding of $V_{1}$.

Let $m_{1}, m_{2}, \ldots, m_{8}$ denote VSP SKIP, Conventional SKIP, Inter16 $\times 16$, Inter16 $\times 8$, Inter $8 \times 16$, Inter $8 \times 8$, Intra16, and Intra4 modes, respectively, where, as before, Inter $8 \times$ 8 includes sub modes Inter8 $\times 4$, Inter $4 \times 8$, and Inter 4 $\times 4$. For a given macroblock in the predicted view $\left(V_{1}\right)$, let $P\left(m_{i} \mid x\right)$ denote the a posteriori probability of selecting mode $m_{i}$ given an observation $x$ of the VSP SKIP RD cost of this macroblock in $V_{1}$. From Bayes theorem, we have $P\left(m_{i} \mid x\right)=P\left(m_{i}\right) p\left(x \mid m_{i}\right) / p(x)$ where $P\left(m_{i}\right)$ is the a priori probability of mode $m_{i}, p\left(x \mid m_{i}\right)$ is the conditional probability density function, and $p(x)$ is the mixture density function. Since $p(x)>0$, Bayes decision rule implies that mode $m_{i}$ should be selected if $P\left(m_{i}\right) p\left(x \mid m_{i}\right)>P\left(m_{j}\right) p\left(x \mid m_{j}\right)$. While the values of $P\left(m_{i}\right)$ and $p\left(x \mid m_{i}\right)$ are unknown in $V_{1}$, we can estimate them from their respective values in $V_{2}$. Fig. 2 shows that the probability density functions $p\left(x \mid m_{i}\right)$ in $V_{1}$ and $V_{2}$ are similar. Here we used a lognormal distribution to model 


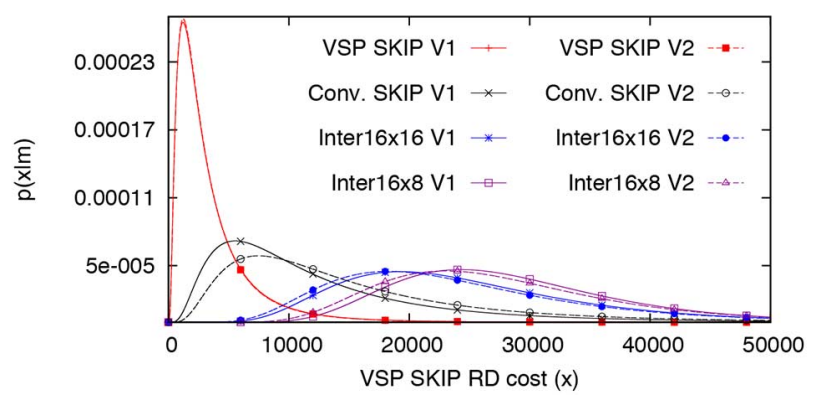

Fig. 2. Comparison of conditional probability density functions (PDFs) in $V_{1}$ and $V_{2}$ for the Breakdancers sequence $(\mathrm{QP}=36)$.

the probability density function of random variable $x$ (Fig. 3). We considered several models and selected the lognormal one based on the Bayesian information criterion [22]. Experimental results also show that the a priori probabilities $P\left(m_{i}\right)$ are similar in $V_{1}$ and $V_{2}$.

Bayes decision rule does not lead to perfect mode selection as its optimality holds in a probabilistic sense only. To account for the Bayes error and the fact that $P\left(m_{i}\right)$ and $p\left(x \mid m_{i}\right)$ are estimates from a different view, we introduce a tolerance threshold $e$ and select not only the optimal mode $m_{i^{*}}$ in Bayesian sense but also any other mode $m_{i}$ such that

$$
\frac{P\left(m_{i^{*}}\right) p\left(x \mid m_{i^{*}}\right)-P\left(m_{i}\right) p\left(x \mid m_{i}\right)}{\sum_{j=1}^{8} P\left(m_{j}\right) p\left(x \mid m_{j}\right)} \leq e
$$

Our algorithm is summarized in Algorithm 1.

Algorithm 1 Bayesian Early Mode Decision Technique

\section{Pre-Processing:}

1: Encode $V_{0}$.

2: Encode $V_{2}$.

Input: Threshold $e$.

Output: Modes to be checked for all macroblocks in $V_{1}$.

1: Calculate the a priori probabilities $P\left(m_{i}\right), i=1, \ldots, 8$ in $V_{2}$.

2: Estimate the conditional probability density functions $p\left(x \mid m_{i}\right)$, $i=1, \ldots, 8$ in $V_{2}$.

3: while not all macroblocks in $V_{1}$ are encoded do

4: Determine the RD cost $x$ of VSP SKIP for the current macroblock in $V_{1}$.

5: Determine $i^{*}=\arg \max _{i} P\left(m_{i}\right) p\left(x \mid m_{i}\right), i=1, \ldots, 8$ from $V_{2}$.

6: for $i=1$ to 8 do

7: $\quad$ if $\frac{\left(P\left(m_{i^{*}}\right) p\left(x \mid m_{i^{*}}\right)-P\left(m_{i}\right) p\left(x \mid m_{i}\right)\right.}{\sum_{j=1}^{8} P\left(m_{j}\right) p\left(x \mid m_{j}\right)} \leq e$ then

8: $\quad$ check mode $i$

9: $\quad$ end if

10: end for

11: end while

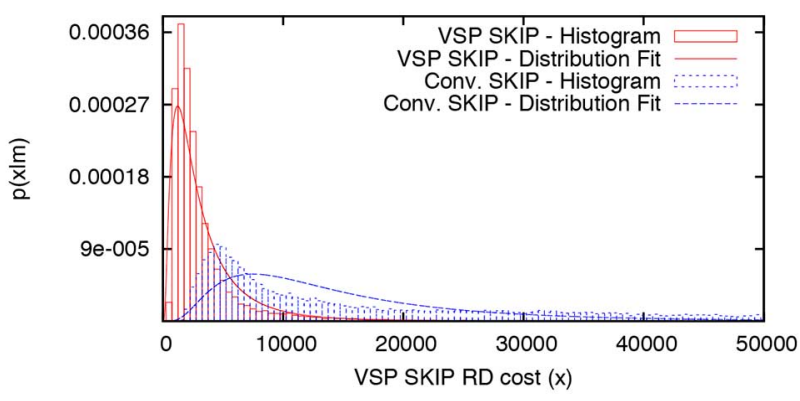

Fig. 3. Normalized histograms of VSP SKIP RD cost for different modes and lognormal distribution fit for the Breakdancers sequence $(\mathrm{QP}=36)$.

TABLE II

Baseline Approach and Proposed Method vs. Standard Coder

\begin{tabular}{|c|c|c|c|c|c|c|c|c|c|}
\hline \multirow[b]{2}{*}{ QP } & \multicolumn{3}{|c|}{ Baseline } & \multicolumn{3}{|c|}{ Proposed $(e=0)$} & \multicolumn{3}{|c|}{ Proposed $(e=0.995)$} \\
\hline & $\triangle \mathrm{PSNR}$ & $\Delta \mathrm{R}(\%)$ & $\Delta \mathrm{T}(\%)$ & $\triangle \mathrm{PSNR}$ & $\Delta \mathrm{R}(\%)$ & $\Delta \mathrm{T}(\%)$ & $\triangle \mathrm{PSNR}$ & $\Delta \mathrm{R}(\%)$ & $\Delta \mathrm{T}(\%)$ \\
\hline & \multicolumn{9}{|c|}{ Breakdancers sequence } \\
\hline 20 & -0.01 & 0.17 & 5.19 & -0.77 & 12.10 & 44.81 & -0.06 & 0.89 & 26.74 \\
\hline 4 & 0.00 & 0.0 & 9.22 & 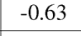 & 14.22 & 46.89 & -0.06 & 0.91 & 28.84 \\
\hline 28 & .01 & 0.0 & 9.57 & 11 & 14.56 & 46.41 & -0.07 & 0.29 & 29.64 \\
\hline 2 & -0.01 & -0.17 & 13.39 & -0.21 & 11.07 & 50.51 & -0.07 & 0.14 & 34.01 \\
\hline 36 & -0.01 & -0.03 & 14.91 & -0.15 & 4.94 & 55.05 & -0.05 & 0.06 & 36.20 \\
\hline \multirow[t]{2}{*}{ Average } & -0.01 & 0.00 & 10.46 & -0.43 & 11.38 & 48.73 & -0.06 & 0.46 & 31.09 \\
\hline & \multicolumn{9}{|c|}{ Ballet sequence } \\
\hline 20 & -0.02 & 0.02 & 9.85 & -0.38 & 12.86 & 48.24 & -0.04 & 0.43 & 22.32 \\
\hline 4 & -0.03 & 0.21 & 20.23 & -0.32 & 13.70 & 49.65 & -0.05 & 0.81 & 25.15 \\
\hline 28 & -0.07 & 1.05 & 27.79 & -0.22 & 12.16 & 50.31 & -0.07 & 0.77 & 27.27 \\
\hline 32 & -0.08 & 0.59 & 28.56 & -0.15 & 9.37 & 50.44 & -0.07 & 0.66 & 33.11 \\
\hline 36 & -0.07 & 0.43 & 27.60 & -0.10 & 4.87 & 47.08 & -0.03 & 0.26 & 35.82 \\
\hline Average & -0.06 & 0.46 & 22.80 & -0.23 & 10.59 & 49.14 & -0.05 & 0.59 & 28.73 \\
\hline Average & -0.03 & 0.23 & 16.63 & -0.33 & 10.98 & 48.93 & -0.06 & 0.52 & 29.91 \\
\hline
\end{tabular}

\section{EXPERIMENTAL RESULTS}

We implemented our fast mode decision technique in the VSP SKIP-enhanced MVC coder described in Section II. As a baseline approach, we used a method that extends the inter-view correlation technique proposed in [13] by selecting a SKIP mode (Conventional SKIP or VSP SKIP) for a macroblock if the corresponding macroblock identified by GDV and its eight neighbouring macroblocks in $V_{2}$ are encoded using the same SKIP mode. The simulations were run on a machine with Intel Core i5 dual core $2.67 \mathrm{GHz}$ CPU and 4 GB RAM.

Table II compares the performance of our technique and the baseline approach to that of the standard coder for $V_{1}$. The encoder settings were as for Table I. Parameters $\triangle \mathrm{PSNR}, \Delta \mathrm{R}$, and $\Delta \mathrm{T}$ denote the increase in PSNR, increase in bitrate, and saving in encoding time, respectively, compared to the standard coder. For our technique, all encoding steps are taken into consideration in the calculation of the encoding time. This includes the estimation of $P\left(m_{i}\right)$ and the fitting of the lognormal distribution model to the samples in $V_{2}$ (Steps 1 and 2 in the main part of Algorithm 1).

The table shows results that correspond to the Bayes decision rule ( $e=0$ in Algorithm 1 ) and to $e=0.995$. For $e=0$, our approach reduced the encoding time by $48.93 \%$ on average. However, the loss in rate-distortion performance was significant. For $e=0.995$, the average saving in encoding time was smaller but still significant $(29.91 \%)$ while the loss in rate-distortion performance was negligible. Further measurements showed that 


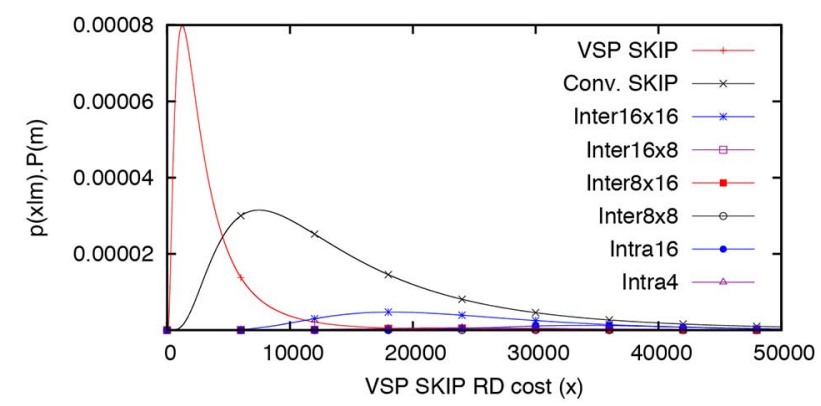

Fig. 4. Product of conditional PDFs and a priori probabilities of different modes for the Breakdancers sequence $(\mathrm{QP}=36)$.

for this value of $e$, our algorithm found the optimal mode for $96.14 \%$ and $92.68 \%$ of the macroblocks for the Breakdancers and Ballet sequence, respectively.

Overall, the time saving for the Breakdancers sequence was slightly greater than for the Ballet sequence because the proportion of VSP SKIP coded macroblocks is higher in the Breakdancers sequence (Table I). The results also show that the reduction in encoding time, generally, increases as QP increases. This is because when QP increases, the quantization becomes coarser, fewer details are preserved, and more large modes are selected. This benefits our method, which mostly predicts large modes such as VSP SKIP, Conventional SKIP, and Inter16 $\times 16$ (Fig. 4).

The baseline approach reduced the encoding time by only $17.4 \%$ on average. Since the synthesized view $S_{2}$ is constructed using only one reference frame, its quality is lower than that of $S_{1}$, which is constructed from two reference frames (Fig. 1). Consequently, the contribution of VSP SKIP in $V_{2}$ is smaller than in $V_{1}$, and the VSP SKIP mode decisions in $V_{1}$ cannot be accurately predicted using the VSP SKIP mode decisions in $V_{2}$.

We obtained similar results for two more test sequences (Poznan_Street and Poznan_Hall2 [21], both of resolution 1920 $\times$ 1088). For example, the average speedup for Poznan_Street and Poznan_Hall2 was $27.03 \%$ and $31.15 \%$, respectively, with negligible loss in rate distortion performance.

\section{CONCLUSION}

We proposed an early mode decision technique for View Synthesis Prediction-enhanced Multiview Video Coding. Our method uses Bayesian decision theory to speed up the encoding by reducing the number of candidate coding modes. Our approach reduced the encoding time of the VSP SKIP-enhanced JMVC 6.0 by up to $36.20 \%$ while preserving the RD performance. We expect that more time savings can be achieved if we combine our method with techniques ([23], [24]) that can efficiently predict non-VSP coding modes. Our results also show that the methods based on inter-view mode correlation might not be suitable for predicting the VSP SKIP mode because of the difference in the quality of the synthesized reference frames in neighbouring views.

\section{REFERENCES}

[1] P. Aflaki, M. Hannuksela, D. Rusanovski, and M. Gabbouj, "Nonlinear depth map resampling for depth-enhanced 3-D video coding," IEEE Signal Process. Lett., vol. 20, no. 1, pp. 87-90, Jan. 2013.
[2] C. Lee and Y. -S. Ho, "A framework of 3D video coding using view synthesis prediction," in Proc. Picture Coding Symposium (PCS'12), Krakow, Poland, May 2012.

[3] S. Yea and A. Vetro, "View synthesis prediction for multiview video coding," Signal Process.-Image Commun., vol. 24, no. 1-2, pp. 89-100, Jan. 2009.

[4] D. Tian, P. Lai, P. Lopez, and C. Gomila, "View-synthesis techniques for 3D Video," in Proc. SPIE 7443, 2009.

[5] Z. Ni, D. Tian, S. Bhagavathy, J. Llach, and B. Manjunath, "Improving the quality of depth image based rendering for 3D video systems," in Proc. IEEE Int. Conf. Image Processing (ICIP'09), Cairo, Egypt, Nov. 2009.

[6] M. Solh and G. Alregib, "Hierarchical hole-filling for depth-based view synthesis in FTV and 3D Video," IEEE J. Sel. Topics Signal Process., vol. 6, no. 5, pp. 495-504, Jun. 2012.

[7] Z.-P. Deng, Y.-L. Chan, K.-B. Jia, C.-H. Fu, and W.-C. Siu, "Iterative search strategy with selective bi-directional prediction for low complexity multiview video coding," J. Vis. Commun. Image Represent., vol. 23, no. 3, pp. 522-534, Feb. 2012.

[8] Y. Zhang, S. Kwong, G. Jiang, X. Wang, and M. Yu, "Statistical early termination model for fast mode decision and reference frame selection in multiview video coding," IEEE Trans. Broadcasting, vol. 58, no. 1, pp. 10-23, Mar. 2012.

[9] S. Khattak, R. Hamzaoui, S. Ahmad, and P. Frossard, "Fast encoding techniques for multiview video coding," Signal Process.-Image Commun., vol. 28, no. 6, pp. 569-580, July 2013.

[10] H. Zeng, K.-K. Ma, and C. Cai, "Fast mode decision for multiview video coding using mode correlation," IEEE Trans. Circuits Syst. Video Technol., vol. 21, no. 11, pp. 1659-1666, Nov. 2011.

[11] L. Shen, Z. Liu, S. Liu, Z. Zhang, and P. An, "Selective disparity estimation and variable size motion estimation based on motion homogeneity for multi-view coding," IEEE Trans. Broadcasting, vol. 55, no. 4, pp. 761-766, Dec. 2009.

[12] L. Shen, Z. Liu, T. Yan, Z. Zhang, and P. An, "View-adaptive motion estimation and disparity estimation for low complexity multiview video coding," IEEE Trans. Circuits Syst.Video Technol., vol. 20, no. 6, pp. 925-930, Jun. 2010.

[13] L. Shen, Z. Liu, T. Yan, Z. Zhang, and P. An, "Early SKIP mode decision for MVC using inter-view correlation," Signal Process.-Image Commun., vol. 25, no. 2, pp. 88-93, Feb. 2010.

[14] H.-S. Koo, Y.-J. Jeon, and B.-M. Jeon, MVC Motion Skip Mode , ISO/IEC JTC1/SC29/WG11 and ITU-T Q6/SG16, Doc. JVT-W081, Apr. 2007.

[15] I. E. G. Richardson, M. Bystrom, and Y. Zhao, "Fast H.264 skip mode selection using an estimation framework," in Poc. Picture Coding Symp. (PCS'06), Beijing, China, Apr. 2006.

[16] C. H. Yeh, K. J. Fan. M. J. Chen, and G. L. Li, "Fast mode decision algorithm for scalable video coding using bayesian theorem detection and markov process," IEEE Trans. Circuits Syst. Video Technol., vol. 20, no. 4, Apr. 2010.

[17] A. Saha, K. Mallick, J. Mukherjee, and S. Sural, "SKIP prediction for fast rate distortion optimization in H.264," IEEE Trans. Consum. Electron., vol. 53, no. 3, pp. 1153-1160, Aug. 2007.

[18] E. Martinian, A. Behrens, J. Xin, and A. Vetro, "View synthesis for multiview video compression," in Proc. Picture Coding Symposium (PCS'06), Beijing, China, Apr. 2006.

[19] Y. Chen, P. Pandit, S. Yea, and C. S. Lim, Draft Reference Software for MVC (JMVC 6.0). London, U.K., Joint Video Team (JVT) Doc. JVT-AE207, Jul. 2009.

[20] L. Zitnick, S. B. Kang, M. Uyttendaele, S. Winder, and R. Szeliski, "High-quality video view interpolation using a layered representation," ACM Trans. Graphics, vol. 23, no. 3, Aug. 2004.

[21] M. Domanski, T. Grajek, K. Klimaszewski, M. Kurc, O. Stankiewicz, J. Stankowski, and K. Wegner, Poznan Multiview Video Test Sequences and Camera Parameters, ISO/IEC JTC1/SC29/WG11 MPEG 2009/M17050. Xian, China, Oct. 2009.

[22] G. Schwarz, "Estimating the dimension of a model," Ann. Statist., vol. 6, no. 2, pp. 461-464, 1978

[23] B. W. Micallef, C. J. Debono, and R. A. Farrugia, "Fast inter-mode decision in multi-view video plus depth coding," in Proc. Picture Coding Symp. (PCS'12), Krakow, Poland, May 2012.

[24] L. Shen, Z. Liu, P. An, R. Ma, and Z. Zhang, "Low complexity mode decision for MVC," IEEE Trans. Circuits Syst. Video Technol., vol. 21, no. 6, pp. 837-843, Jun. 2011. 